\title{
Male sterility in triploid dandelions: asexual females vs asexual hermaphrodites
}

\author{
PG Meirmans ${ }^{1,2}, \mathrm{H}(\mathrm{J}) \mathrm{CM}$ Den Nijs ${ }^{1}$ and PH Van Tienderen ${ }^{1}$ \\ ${ }^{1}$ Institute for Biodiversity and Ecosystem Dynamics, Kruislaan 318, 1098 SM, Amsterdam, The Netherlands; ${ }^{2}$ Laurentian Forest Center, \\ Canadian Forest Service, 1055 Rue du PEPS, Sainte-Foy, Québec, Canada G1V 4C7
}

\begin{abstract}
Male reproductive output, pollen in plants and sperm in animals has been shown to constitute a substantial cost for many organisms. In parthenogenetic hermaphrodites, selection is therefore expected to reduce the allocation of resources to male reproductive output. However, sustained production of pollen or sperm has been observed in numerous asexual hermaphrodites. We studied the widespread production of pollen by triploid asexual dandelions, Taraxacum sect. Ruderalia, comparing rare male sterile individuals with pollen producing asexuals. We found that individuals can show plasticity in the production of pollen, but that it is nevertheless possible to distinguish between (facultatively) male sterile asexuals and male fertile asexuals. Based on evidence from genetic markers and
\end{abstract}

crosses, we conclude that the male sterility in asexual dandelions is caused by nuclear genes, in contrast to the cytoplasmically inherited male sterility previously found in sexual dandelions. Male sterile lineages did not produce more seeds per flower head, heavier seeds or seeds that were more viable. However, male sterile plants did produce more seed heads and hence more seeds than pollen producing ones, indicating that they were able to reallocate resources toward seed production. Considering the difference in seed production, it remains puzzling that not more asexual dandelions are male sterile.

Heredity (2006) 96, 45-52. doi:10.1038/sj.hdy.6800750; published online 28 September 2005

Keywords: apomixes; polyploidy; parthenogenesis; Taraxacum; sex allocation

\section{Introduction}

Male reproductive output, pollen in plants and sperm in animals, has often been found to be costly (Gouyon and Couvet, 1987). As a result of this, in parthenogenetically reproducing hermaphrodites, male reproductive output is expected to be eliminated through natural selection (Weinzierl et al, 1998). Nevertheless, numerous asexual hermaphrodites maintain their male function; marine clams (Ó Foighil and Smith, 1995) and flatworms (Weinzierl et al, 1998) produce sperm, blackberries (Kollmann et al, 2000) and most dandelions (Richards, 1986) produce pollen. A nonadaptive explanation for the retention of the male function of such asexual hermaphrodites has been given by Maynard Smith (1978), who suggested that the asexual lineages in these species may be too young and may not yet have had the time to accumulate the necessary mutations for male sterility. Alternatively, male function could be retained selectively, if pollen or sperm are used to fertilise sexual relatives and if such matings lead to the production of new clonal lineages or reduce the fitness of sexual competitors. Male function can also be adaptive in pseudogamous species, where parthenogens require sperm or pollen to trigger embryo development (Weinzierl et al, 1998).

The production of pollen is widespread among asexually reproducing forms of common dandelions,

Correspondence: PG Meirmans, Laurentian Forest Center, Canadian Forest Service, 1055 Rue du PEPS, Sainte-Foy, Québec, Canada G1V 4C7. E-mail:pmeirmans@nrcan.gc.ca

Received 11 November 2004; accepted 22 July 2005; published online 28 September 2005
Taraxacum sect. Ruderalia (Richards, 1986). This pollen production has been seen as a pure waste of resources (Maynard Smith, 1978; Van Dijk, 2003) since asexual dandelions do not require pollination to trigger endosperm development. Furthermore, asexual dandelions are predominantly triploid and, therefore, as a result of unbalanced meiosis, most of the produced pollen is inviable. Sexual dandelions are diploid and, due to a sporophytic self-incompatibility system, outcrossing. There is a clear geographic pattern in the distribution of the sexuals and asexuals; the asexuals have a much larger and more Northern distribution than the sexuals, which are confined to Southern and Central Europe, where the two types are often found together in mixed populations (Den Nijs and Sterk, 1980, 1984). The occurrence of both sexuals and asexuals complicates the taxonomy of the genus Taraxacum to a large extent. The genus is generally divided into sections rather than species, with sections being loose collections of dandelion taxa sharing morphological traits. Genetic analysis showed that those sections are not monophyletic groups; a large part of the chloroplast variation was shared between the sections (Wittzell, 1999; Kirschner et al, 2003). This study focuses on section Ruderalia, which is the most widespread section and includes the majority of dandelions found in central Europe.

Even though the great majority of dandelions of section Ruderalia (hereafter simply referred to as 'dandelions') produce pollen, male sterility has been found in low frequencies among triploid asexual but also among diploid sexual dandelions. Among the asexuals, male sterility is present all over Europe but usually only at low 
frequencies. From observations of a large number of populations of asexual dandelions from Western Europe (sampling about 30 asexual individuals per population), we estimate the overall frequency of male sterility to be between 1 and 5\% (PG Meirmans, pers. obs.).

In diploid sexual dandelions, male sterility was found in two populations from France by Van der Hulst et al (2004), who proved through crosses that the trait is cytoplasmically inherited, and that pollen production can be restored by nuclear modifying genes. The results from the crosses were corroborated by linkage between male sterility and a rare chloroplast haplotype. This haplotype was present in all male sterile sexuals from two populations and further in only one male fertile individual, in which pollen production was probably restored by (a) nuclear modifier(s). The male sterility in sexual dandelions is most likely caused by a mutation on the mitochondrial DNA, as this has often been observed in angiosperms (Hanson, 1991). The linkage between the chloroplast haplotype and the male sterility trait is then the result of the strong linkage that exists between the mitochondrial and chloroplast genomes because both are strictly maternally inherited in Asteraceae (Corriveau and Coleman, 1988).

Male sterile polyploid asexuals can be generated through crosses between a male sterile diploid sexual mother and triploid asexual pollen donors (Van der Hulst et al, 2004). If there really were resource reallocation due to male sterility, these new male sterile asexuals would have an immediate fitness benefit over pollen producing asexuals. As triploid asexual plants sometimes pollinate diploid sexual plants under field conditions (Sterk, 1987; Menken et al, 1995; Meirmans et al, 2003), pollinations of male sterile sexuals by male fertile asexuals could result in the rapid generation of new male sterile asexual lineages. However, even though the populations in which the male sterile sexuals were found were mixed sexual/asexual populations, no male sterile asexuals and no asexuals containing the rare chloroplast haplotype were found in the populations containing male sterile sexuals (Van der Hulst et al, 2004).

Pollination of sexuals by asexuals is possible because not all pollen that is produced by the triploid asexuals is inviable. Crosses between sexuals and asexuals generally result in diploid, triploid and tetraploid offspring, though the latter are very rare in natural populations (Verduijn et al, 2004). Hybridisations between sexuals and asexuals are believed to be relatively common in natural populations as most variation at allozyme and microsatellite loci is shared between the sexuals and the asexuals (Menken et al, 1995; Meirmans et al, 2003). Owing to the possibility of such hybridisations, the fitness benefit of male sterility in asexuals due to resource reallocation towards the female function could be balanced by the fitness benefit through the pollination of the sexuals. Provided that there is not much phenotypic plasticity in the expression of pollen production, new clonal lineages that are created through this process will most likely be male fertile. Therefore, the presence of male sterility in dandelions, as well as other asexual hermaphrodites, is not only determined by the rate of mutation to male sterility, but also by the fitness trade-off between resource reallocation and rate of gene flow with the sexuals (Weinzierl et al, 1998). There is however little information on the importance of these different processes in any asexual hermaphrodite.

\section{Research goals}

The aim of this research is to answer the following questions regarding the evolution of male sterility in asexual dandelions: (1) Is there any plasticity in pollen production; that is, do all capitula that are produced by an individual have the same phenotype? (2) Is there a genetic basis for male sterility in asexuals, and if so, is it cytoplasmically inherited and linked to the same chloroplast haplotype as in the male sterile sexuals (Van der Hulst et al, 2004)? (3) Does male sterility provide individual plants with a fitness benefit due to resource reallocation? (4) Are pollen producing clones more related to the sexuals than male sterile clones, which is expected considering that most gene flow between sexuals and asexuals is thought to occur through pollen produced by asexuals?

We answer these questions by using chloroplast haplotyping and microsatellite genotyping, crosses between (partially) male sterile asexuals and sexuals, measuring reproductive characters, and by estimating the amount of genetic differentiation by comparing microsatellite data of asexuals and sexuals.

\section{Materials and methods}

\section{Population sampling}

In 2001, three dandelion populations with coexisting sexual diploids and asexual triploids were sampled from Brno, Kyov and Znojmo (Czech republic) as a part of a phylogeographic study. These three population samples were chosen for the present study from more than 30 available samples in our greenhouse because of the high frequency of male sterility among the asexuals in these populations. Sampling was performed by Den Nijs and Kirschner (who confirmed that all sampled plants belonged to Taraxacum section Ruderalia). The dandelions were dug out, leaves were removed and the roots were transferred to the greenhouse in Amsterdam, where the plants were regrown. We scored the ploidy level and the mode of reproduction of the plants using four methods: (1) Size variation in the pollen grains (only possible with male fertile plants): triploid plants have a disturbed meiosis and produce pollen of different sizes, whereas diploids produce regularly sized pollen. (2) Spontaneous seed production: insect pollinations are very rare in our greenhouse so nearly all capitula that develop seeds are from asexual plants. (3) Number of alleles at four microsatellite loci. (4) Flow cytometry, using a Partec Ploidy Analyser, according to the instructions of the manufacturer. The latter method was used to score the ploidy level of the male sterile plants and the offspring from the crosses.

\section{Scoring of male sterility}

During the flowering seasons of 2002 and 2003, all capitula of all individuals of the three Czech populations were scored for pollen production. This was done by looking at the open capitula with a $\times 10$ magnifying glass to see whether the anther tube and stamen of the open florets contained pollen. Capitula were scored as male fertile, male sterile and partially male sterile. 
Capitula were assigned to the latter category when they contained mostly male sterile but also some male fertile florets. Based on the produced capitula, we assigned individuals as being male sterile or male fertile (see Results).

\section{Genotyping}

DNA-extraction, microsatellite-amplification and chloroplast PCR-RFLP were all performed using the protocols described in Van der Hulst et al (2003). Four microsatellite loci were used for genotyping the plants: msta64, msta72, msta78 and msta85 (Falque et al, 1998). Microsatellite amplification products were run on polyacrylamide on a Li-Cor GeneReadir 4200 sequencer. The banding patterns were scored by hand twice; inconsistencies were scored a third time. Whenever triploid individuals only had two bands at a locus, the relative dosages of the two bands were used to score which allele was present once and which was present twice. Dosage effects were clear in most of these cases; in the few cases that were ambiguous, the third allele was scored as 'missing data'. Three regions of the chloroplast genome, known to contain length and restriction-site variation in dandelions (Van der Hulst et al, 2003), were amplified using universal primers: intron trnL (Taberlet et al, 1991) cut with Bste2, trnL-trnF (Taberlet et al, 1991) and psbAtrnH (Dumolin-Lapeque et al, 1997) cut with Dra1 and Ssp1. All chloroplast variation was scored from agarose gels, except the length variation at the psbA-trnH intergenic spacer, for which labeled primers were used and which was run on the Li-Cor.

\section{Crosses}

We performed crosses to assess whether the inheritance of the male sterility trait in the asexuals is nuclear or cytoplasmic, even though it is rather difficult to do crosses with male sterile apomicts. When a partially male sterile capitulum was discovered on an individual that was otherwise fully male sterile, this capitulum was immediately used to pollinate a sexual individual. In most of these crosses, the sexuals failed to set seed, but a small number of viable seeds was produced in three of the trials, involving three father plants from three different clonal lineages, and three mothers. All seeds produced from the crosses were put in the climate chamber for germination under the same conditions as described below. It is known that pollination of a diploid sexual motherplant with pollen from a triploid asexual can result in breakdown of the self-incompatibility system in the sexual (Morita et al, 1990); it is, therefore, important to check for selfing of the mother plants. We did this through genotyping the parents and all the offspring that survived to maturity at three microsatellite loci: msta53, msta72 and msta78 (Falque et al, 1998). All plants of the progeny that came out as hybrids were subsequently checked for male sterility when they flowered.

\section{Reallocation of resources}

To test whether male sterility provides plants with more resources for the production of more and/or better seeds, we measured a number of reproductive traits. In 2002 and 2003, all seed heads were collected from the asexual plants, recording the pollen phenotype of the capitulum when it flowered. We also counted, weighed and germinated seeds produced by 22 male sterile and 29 male fertile plants during the flowering season of 2002. For this, depending on the number of produced seed heads, either one or two heads were used per plant, and if a plant had produced more than two seed heads, two heads were chosen at random. For each seed head, we counted the total number of seeds as well the number of inviable seeds; these are easily recognisable because they are thin and white, in contrast to the normal bigger, brown seeds. In total, seeds, including the pappus, were weighed per seed head. To control for the possibility that, unconsciously, the bigger seeds were picked first, we performed the weighting in two sets of 30 seeds each, allowing us to check whether the first 30 seeds were heavier than the second batch. These same sixty seeds were used for the germination experiments that were performed in two rounds, each round using one of the two sets of 30 seeds. Whether the first or second batch of 30 seeds was used for the first or second germination round was determined at random, by flipping a coin. For the germination experiments, the 30 seeds were placed in a titerplate containing wet filterpaper and all titerplates were placed in a climate chamber with an $18 / 14^{\circ} \mathrm{C}$ temperature and a $16 / 8 \mathrm{~h}$ dark/light regime. The titerplates were checked daily for 4 weeks; newly germinated seedlings were counted and removed. No differences were found between the first and second set of 30 seeds that were picked per seed head, nor were there any differences between the first and second round of germination, so the results were combined.

\section{ANOVAs}

Seed and germination characteristics were analysed using ANOVAs. Number of seed heads and percentage of inviable seeds were log-transformed, and the percentage of seeds that germinated was arcsine-transformed prior to analysis. The number of seed heads was analysed with male sterility (sterile $v$ s fertile), population and year as fixed factors and genotype nested within sterility by population as random factor. The number of seeds within seed heads, percentage of sterile seeds, seed weight and germination rate were analysed with sex type and population as fixed factors, and individual nested within genotype nested within sterility by population as random factors. ANOVAs were performed using SPSS version 11.

\section{Clone assignment}

Multilocus microsatellite genotypes were used to assign asexual individuals to clonal lineages. If such assignment is carried out based on full identity in multilocus genotype, there may be an error as, due to mutations or PCR-artefacts, individuals from the same clonal lineage may have slightly different microsatellite profiles. Therefore, to assign individuals to clones we used a threshold: the maximum pairwise genetic distance allowed between individuals to belong to the same clonal lineage (Douhovnikoff and Dodd, 2003; Meirmans and Van Tienderen, 2004). This was carried out using the frequency distribution of pairwise distances between individuals. For asexual organisms such a frequency distribution often shows two peaks: a peak close to zero 
representing comparisons between clonemates and a second peak representing comparisons between individuals belonging to different clones. The 'valley' between the two peaks is then a suitable candidate for the threshold that is used for assigning individuals to clonal lineages. This assignment was carried out using the program GenoType (Meirmans and Van Tienderen, 2004). As a distance measure, we used the number of single basepair length mutations that is needed to transform one genotype into the other. Association between the clonal lineages (hereafter called 'clones') and male sterility, and between the chloroplast haplotypes and male sterility was carried out using the log likelihood G-statistic, the significance of which was assessed through 999 random permutations of the data, using the program GenoDive (Meirmans and Van Tienderen, 2004). This program was also used to test for differences between the populations in the frequencies of clones and cp-haplotypes.

\section{Genetic differentiation}

Gene flow between sexual and asexual dandelions is thought to occur mostly due to pollinations of sexuals by pollen from asexuals. Therefore, pollen producing asexuals are expected to be genetically more similar to the sexuals than male sterile asexuals. We tested this hypothesis using the Rho-statistic (Ronfort et al, 1998), an $\mathrm{F}_{\mathrm{st}}$-like estimator for the amount of population divergence, based on allele frequencies, that is suited for comparisons involving different ploidy levels. Per population, we estimated the Rho-statistic between the sexuals, the male sterile and male fertile asexuals. For an overall estimate, we averaged the three withinpopulation estimates. To test the hypothesis of higher divergence of the male sterile asexuals, we took a bootstrapping approach: we calculated a test statistic by subtracting the averaged Rho-estimate of the divergence between the sexuals and the male sterile asexuals from the estimate of the divergence between the sexuals and the male fertile asexuals. We then created resampled data sets, through bootstrapping within populations, recalculated the test statistic and compared this bootstrapped value to the original value (Manly, 1991). As this was a very time-consuming approach, only 20 of such resampled data sets were created. The calculations of the Rho-statistic were done using the program Spagedi (Hardy and Vekemans, 2002).

\section{Results}

\section{The presence of male sterility}

Among the 178 plants analysed, 75 were diploid sexuals, 102 were triploid asexuals and one was a tetraploid asexual. Male sterile capitula were produced by in total 24 plants, all of which were asexual triploids. Partially male sterile capitula were only produced by plants that also produced male sterile capitula; all other plants produced only fully male fertile capitula. Among the 24 plants that showed male sterility, there were three individuals that also produced some fully male fertile capitula, but for all three individuals these fully male fertile capitula accounted for less than $20 \%$ of the total number of produced capitula. It was therefore possible to make a clear distinction between plants that only produced male fertile capitula and those that predominantly produced male sterile capitula but occasionally produced capitula with pollen (for brevity we will refer to the latter group as 'male sterile' rather than 'facultatively male sterile'). The frequency of the male sterile plants differed between the populations, with male sterile plants comprising $35 \%$ of the asexuals at Brno, $27 \%$ at Znojmo and $12 \%$ at Kyov.

\section{Clones, haplotypes and male sterility}

The frequency distribution of pairwise genetic distances between individuals, based on four microsatellite loci, showed two distinct peaks (webmaterial Figure 1). Based on this frequency distribution we chose a threshold of six mutation steps for assigning individuals to clones. Using this method, 45 different clones were distinguished among the asexuals, 28 of which were found only once. Within the clones that were found more than once, all members typically belonged to the same pollen type (Table 1). The only exception to this was one member of clone number five that repeatedly produced pollen, while the other seven members of the same clone were typed as male sterile. Interestingly, one of the three plants that produced both male sterile and male fertile capitula also belonged to this clone. The association between clone and male sterility was strongly significant (999 permutations, $P=0.001$ ). In contrast, there was no association between male sterility and the seven chloroplast haplotypes that were found (Table 2); the chloroplast haplotype associated with male sterility in diploid sexuals (Van der Hulst et al, 2004) was not found in the studied populations. The results from the microsatellite and chloroplast analyses therefore suggest nuclear rather than cytoplasmic inheritance of male sterility in the polyploid asexuals.

\section{Crosses}

To further study the inheritance of male sterility in asexual dandelions, we made crosses between (facultatively) male sterile triploid pollen donors and male fertile sexual diploids. The three successful crosses, out of more

Table 1 Clone frequencies in male fertile male and sterile asexuals dandelions

\begin{tabular}{lcc}
\hline Clone $n r$. & Male fertile & Male sterile \\
\hline 1 & 10 & 2 \\
3 & & 3 \\
4 & 1 & 7 \\
5 & 2 & 2 \\
10 & 13 & 2 \\
15 & & \\
20 & 2 & \\
23 & 2 & \\
25 & 3 & \\
31 & 5 & \\
32 & 2 & \\
33 & 2 & \\
34 & 3 & 24 \\
37 & 3 & \\
41 & 22 & \\
42 & & \\
43 & 70 & \\
Unique & & \\
Total & & \\
\hline
\end{tabular}


Table 2 Chloroplast haplotype frequencies in male fertile asexual, male sterile asexual and sexual dandelions

\begin{tabular}{lccc}
\hline Haplotype & Sexual & MS asexual & MF asexual \\
\hline 1 & 28 & 22 & 53 \\
2 & 25 & 1 & 14 \\
3 & 1 & 1 & 3 \\
4 & 3 & & 1 \\
5 & 1 & & 1 \\
6 & 2 & 24 & 72 \\
Total & 60 & 24 & \\
\hline
\end{tabular}

than 20 attempts, yielded very low seed set: 5, 17 and 76 viable brown seeds were produced, of which, respectively, 5, 7 and 25 seeds germinated. Microsatellite analysis showed that part of the progeny had resulted from selfing ( 0,1 and 23 respectively), so that the three crosses resulted in $13 \mathrm{~F}_{1}$ hybrids: eight diploid, three triploid and two tetraploid individuals. Two of the three crosses indicated nuclear inheritance of male sterility as some of the offspring were male sterile, apparently inherited from their father: in the first cross two diploids and one triploid and in the second cross four diploids were male sterile. The data from the third cross were inconclusive as the only two plants that resulted from the cross were both tetraploid and male fertile. The number of offspring in the crosses was too low to perform any test for Mendelian inheritance of the male sterility trait.

\section{Reproductive characters}

Male sterile clones produced more seed heads than pollen producing ones. However, seeds produced by male sterile clones were lighter rather than heavier compared to seeds of male fertile clones. There was no difference between male sterile and male fertile clones in the number of seeds per head, the percentage of inviable seeds and the germination rate (Tables 3 and 4). There were no differences among the three populations for all measured variables, nor were there any interaction effects. There were also no differences between years or among clones nested within male sterility/population. Individuals nested within clones showed differences for seed weight and germination rate.

\section{Genetic differentiation}

The male sterile asexuals were genetically not more differentiated from the sexuals than the male fertile asexuals. The multilocus Rho estimate for the divergence between the sexuals and the male sterile asexuals $($ Rho $=0.137)$ was only slightly higher than that between the sexuals and the male fertile asexuals $(\mathrm{Rho}=0.135)$. This small difference between the two Rho estimates proved not significant when tested using bootstrapping $(P=0.67)$. However, both Rho estimates were significantly different from zero (1000 permutations, $P=0.001$, for both values), indicating that both the male sterile and the pollen producing asexuals were genetically differentiated from the sexuals. Nevertheless, the relatively low value of the Rho-statistic indicates that most of the genetic variation was shared between the sexual and asexual populations.
Table 3 Seed and germination characteristics of male sterile and male fertile dandelions

\begin{tabular}{lrr}
\hline & Male fertile & Male sterile \\
\hline \# of heads, 2002 & $3.03(0.35)$ & $4.3(0.94)$ \\
\# of heads, 2003 & $7.45(1.41)$ & $9.99(2.21)$ \\
Seed weight $(\mathrm{mg})$ & $5.9(0.25)$ & $4.92(0.53)$ \\
\# of seeds per head & $173.56(23.1)$ & $179.38(2.77)$ \\
\% inviable seeds & $8.84(5.33)$ & $7.43(1.69)$ \\
\% germinated & $74.68(4.04)$ & $61.99(6.28)$ \\
\hline
\end{tabular}

Values are averaged over populations, with genotypes nested within populations and individuals within genotypes. Standard deviation over populations is indicated in parentheses.

\section{Discussion}

Facultative male sterility

It is clear from our results that there is plasticity in the expression of male sterility in triploid asexual dandelions: more than half of the plants that produced male sterile capitula also produced some partially male sterile capitula (capitula with a small number of pollen producing florets). Three of the plants that were typed as facultatively male sterile produced some fully male fertile capitula. The plasticity in pollen production in the asexual dandelions was asymmetric: there were individuals that predominantly produced male sterile capitula and also produced some capitula with pollen, but there were no individuals that predominantly produced male fertile capitula with a low number of male sterile capitula. This asymmetry, together with the results from the crosses and the strong association between clones and male sterility, shows that in asexual dandelions, despite the found plasticity, male sterility is a clearly delimited trait with a genetic basis.

Plasticity in pollen production has previously been noted in the offspring of crosses between male sterile diploid sexual dandelions and triploid asexual pollen donors, where the production of some individuals depended on the temperature 2 weeks before anthesis (Van der Hulst et al, 2004). It is possible that such temperature dependence is also present in the plants under study here, but as we do not have temperature data for the 2 years in which we followed the pollen production, we cannot test this.

\section{Mode of inheritance}

The results of the crosses, the microsatellites and the chloroplast haplotyping all indicate that the inheritance of male sterility in these populations of asexual dandelions has a nuclear basis. First, there was a close correspondence between multilocus microsatellite genotype and male sterility. Second, the PCR-RFLPs showed no correspondence between the chloroplast haplotype and male sterility; furthermore, the chloroplast haplotype that is indicative for a male sterility inducing cytoplasm (Van der Hulst et al, 2004) was not found in the populations studied here. Third, the crosses between sexual dandelions and predominantly male sterile asexuals resulted in male sterile offspring in two out of the three crosses.

The nuclear inheritance of male sterility in asexual dandelions found in our study does not match the 
Table 4 ANOVAs, comparing seed and germination characteristics between male sterile and male fertile dandelions (MS), between populations (POP) and years (YEAR), with genotypes (CLONE) nested within MS by POP and with individuals (IND) nested within genotypes

\begin{tabular}{|c|c|c|c|c|c|}
\hline & Mean sq. & Err. mean sq. & d.f. & $F$ & $\mathrm{P}$ \\
\hline \multicolumn{6}{|l|}{ Number of seed heads produced } \\
\hline MS & 0.55 & 0.02 & $1 / 1.4$ & 47.66 & 0.045 \\
\hline POP & 0.02 & 0.31 & $2 / 2.4$ & 0.09 & 0.915 \\
\hline YEAR & 2.29 & 0.16 & $1 / 1.6$ & 22.94 & 0.065 \\
\hline $\mathrm{MS} * \mathrm{POP}$ & 0.00 & 0.09 & $2 / 3.3$ & 0.04 & 0.959 \\
\hline MS*YEAR & 0.00 & 0.03 & $1 / 2.2$ & 0.03 & 0.877 \\
\hline POP*YEAR & 0.24 & 0.02 & $2 / 1.3$ & 9.36 & 0.167 \\
\hline $\mathrm{MS} * \mathrm{POP} * \mathrm{YEAR}$ & 0.03 & 4.45 & $2 / 79.2$ & 0.26 & 0.770 \\
\hline GENOTYPE(MS*POP) & 3.23 & 2.43 & $47 / 46.3$ & 1.31 & 0.180 \\
\hline GENOTYPE(MS*POP*YEAR) & 2.23 & 4.99 & $43 / 72$ & 0.75 & 0.844 \\
\hline \multicolumn{6}{|l|}{ Seed weight } \\
\hline MS ${ }^{\circ}$ & 16598.9 & 3961.4 & $1 / 29.3$ & 4.19 & 0.049 \\
\hline POP & 2616.2 & 3973.5 & $2 / 28.8$ & 0.66 & 0.525 \\
\hline $\mathrm{MS} * \mathrm{POP}$ & 926.1 & 3983.9 & $2 / 28.9$ & 0.23 & 0.794 \\
\hline CLONE (MS*POP) & 3951 & 2510.6 & $30 / 14.4$ & 1.57 & 0.182 \\
\hline IND (CLONE (MS*POP)) & 2381.8 & 687.2 & $15 / 40$ & 3.47 & 0.001 \\
\hline \multicolumn{6}{|l|}{ Number of seeds per head } \\
\hline MS & 790.5 & 4335.5 & $1 / 29.6$ & 0.18 & 0.672 \\
\hline POP & 7957.2 & 4329.4 & $2 / 28.7$ & 1.84 & 0.177 \\
\hline $\mathrm{MS} * \mathrm{POP}$ & 6173 & 4338.9 & $2 / 28.9$ & 1.42 & 0.257 \\
\hline CLONE (MS*POP) & 4347.6 & 4307.9 & $30 / 15.8$ & 1.01 & 0.510 \\
\hline IND (CLONE (MS*POP)) & 4282.1 & 2310.4 & $16 / 45$ & 1.85 & 0.053 \\
\hline \multicolumn{6}{|l|}{$\%$ inviable seeds } \\
\hline MS & 0.000 & 0.231 & $1 / 29.6$ & 0.00 & 0.983 \\
\hline POP & 0.233 & 0.233 & $2 / 29.3$ & 1.00 & 0.380 \\
\hline $\mathrm{MS} * \mathrm{POP}$ & 0.030 & 0.233 & $2 / 29.3$ & 0.13 & 0.879 \\
\hline CLONE (MS*POP) & 0.229 & 0.096 & $30 / 13.3$ & 2.39 & 0.047 \\
\hline IND (CLONE (MS*POP)) & 0.095 & 0.078 & $15 / 40$ & 1.21 & 0.306 \\
\hline \multicolumn{6}{|l|}{$\%$ germinated } \\
\hline MS & 0.648 & 0.222 & $1 / 29.2$ & 2.92 & 0.098 \\
\hline POP & 0.022 & 0.222 & $2 / 28.5$ & 0.10 & 0.907 \\
\hline $\mathrm{MS} * \mathrm{POP}$ & 0.168 & 0.223 & 2/28.6 & 0.76 & 0.479 \\
\hline CLONE (MS*POP) & 0.221 & 0.178 & $30 / 14.3$ & 1.24 & 0.341 \\
\hline IND (CLONE (MS*POP)) & 0.172 & 0.07 & $15 / 39$ & 2.47 & 0.012 \\
\hline
\end{tabular}

Number of seed heads and percentage of inviable seeds were log-transformed; the percentage of seeds that germinated was arcsinetransformed prior to analysis.

findings of Malecka (1971), who argued, based on anther morphology, that male sterility in two asexual dandelion lineages was cytoplasmic. She argued that the pattern of the tapetal layer degeneration was characteristic for cytoplasmic male sterility. Whereas we can exclude cytoplasmic inheritance in two of our crosses, where male sterile offspring was obtained, it cannot be excluded in the third; the offspring of hybrid origin of this cross were two pollen-producing individuals. These two plants were both tetraploid and therefore presumably received the complete nuclear genome of their male sterile triploid father and a haploid set from their mother. As the offspring were producing pollen, the male sterility of the father may have been cytoplasmic and not transmitted to the offspring. Alternatively, the male sterility may have been restored by alleles present in the mother plant.

\section{Fitness benefit of male sterility}

The male sterile dandelions in the studied populations reallocated resources into the production of more seed heads. Averaged over the 2 years that the production of seed heads was monitored, the male sterile plants produced $38 \%$ more seed heads than the pollen producing ones. Seeds produced by male sterile clones were lighter than those of pollen producing clones, but, as we found no difference in germination rate, seed weight might have little effect on viability. The increased seed production of the male sterile clones therefore gives them a large fitness benefit that makes the widespread occurrence of pollen production in asexual dandelions particularly puzzling. Apart from any nonequilibrium scenarios in which male sterility simply did not yet go to fixation, there are two explanations for why most asexuals produce pollen. The first explanation is that the produced pollen gives the male fertile asexuals a fitness benefit through the pollination of coexisting sexuals. The second explanation is that balancing selection maintains clonal diversity, which includes diversity in pollen production.

It is known that the pollen produced by asexuals, even though it is largely inviable, can occasionally pollinate sexual diploid dandelions, resulting in gene flow between the sexuals and asexuals (Verduijn et al, 2004). The occurrence of gene flow between sexuals and 
asexuals is apparent in the present study from the low values of the Rho statistic (which is like $F_{\text {st }}$ bound between 0 and 1). Such gene flow leads to the creation of new asexual lineages, and therefore the production of pollen may provide a fitness benefit for pollen producing asexuals. In this study, we found that male sterile asexuals have a $38 \%$ higher reproductive output than pollen producing asexuals. If male sterile and pollen producing asexuals have equal germination and survival in the field environment, the male sterile asexuals have a $38 \%$ higher fitness. It seems unlikely to us that hybridisations between sexuals and pollen producing asexuals are frequent enough to balance this fitness difference. To reach this balance, the effective rate of production of new clonal lineages through pollen produced by asexuals has to be equal to the same $38 \%$ per generation. In a study of a dandelion population containing both sexuals and asexuals, Verduijn et al (2004) found that less than $2 \%$ of the offspring produced by sexuals was polyploid. Even if newly produced clonal lineages have a fitness benefit compared to the existing lineages (eg due to purging of deleterious mutations or escape from specialised parasites), the rate of gene flow required to balance the fitness benefit of male sterility would have to be unrealistically high, especially when considering the finding that newly created clones had a much lower seed set than established clones (De Kovel and De Jong, 2000).

Balancing selection can maintain variation in pollen production among clonal lineages if it is not pollen production in itself but clonally linked traits that are under selection. Under the Frozen Niche Variation Hypothesis (Vrijenhoek, 1979), clonal lineages coexist in separate ecological niches. Under such a scenario, the male sterile lineages may have a higher production of seed heads, but may still not be able to outcompete the pollen producing lineages in their respective ecological niches. In dandelions, niche differentiation between two clonal lineages has been shown by Solbrig and Simpson (1974), who showed that one of the two clones was better at competition, while the other had a higher seed set and was therefore assumed to be better at colonisation. Meirmans (2005) found that although clonal lineages differed from each other in seed production, they did not differ in their habitat, measured by the vegetation growing around the individuals. It is therefore uncertain whether the high clonal diversity that is generally found in dandelions (Van der Hulst et al, 2003) is maintained by Frozen Niche Variation.

\section{Genetic differentiation}

The observed lack of a difference in relatedness with the sexuals between the male sterile asexuals and the male fertile asexuals can be explained by the plasticity in pollen production of the (facultatively) male sterile asexuals. The crosses between the sexuals and male sterile asexuals indicate that the latter can successfully act as pollen donors, though only few of the crosses were successful. Gene flow between male sterile asexuals and sexuals is therefore possible and repeated production of new male sterile clones would prevent a high amount of genetic differentiation between the male sterile asexuals and the sexuals. However, considering the difference in the amount of pollen produced by the facultative male sterile asexuals and the male fertile asexuals, a difference in the rate of gene flow with the sexuals is expected. It is however doubtful whether such a relatively small difference would be detectable using the Rho-statistic, which, like all $\mathrm{F}_{\text {st-analogues, has a relatively high }}$ associated standard error, especially with low sample sizes such as used in this study.

Limited gene flow between sexuals and male sterile asexuals even does not exclude the possibility of a single mutational origin of the male sterility trait in the studied populations, despite the fact that male sterility was found in individuals with 12 different microsatellite genotypes and three different chloroplast haplotypes. However, we think that independent evolutionary origins and genetic causes are more likely; we observed that there was variation in the morphology of male sterile capitula in different individuals as in some male sterile individuals the capitula had distinctly brown coloured anther tubes, while in the other male sterile individuals the capitula had yellow anther tubes (PG Meirmans, pers. obs.).

\section{Conclusions}

Male reproductive output of parthenogenetic hermaphrodites is determined by the trade off between the reallocation of resources into the female function, leading to male sterility, and the ability of the male gametes to fertilise sexual relatives, leading to the production of new clones. Furthermore, the evolution of male sterility in asexuals is constrained by the rate of mutation to male sterility. However, the argument that there has not been enough time for the asexuals to accumulate the necessary mutations for male sterility (Maynard Smith, 1978) does not hold for the dandelions under study here. The mutations have occurred, possibly even several times, but they did not go to fixation, despite a substantial fitness benefit due to the reallocation of resources. This fitness benefit and the observation that male sterility is present in several different clonal lineages should give the male sterile dandelions high evolutionary potential, which makes the widespread occurrence of pollen producing asexual dandelions seem quite paradoxical.

\section{Acknowledgements}

This work would not have been possible without the help of Louis Lie, who counted, weighted and germinated the seeds. We also thank Jan Kirschner for help in sampling and identifying the dandelions, Ludek Tikovsky for taking care of the plants in the greenhouse, Rob Bregman for help in collecting the seeds and Peter Kuperus for assistance in the lab. We thank Stephanie Meirmans and two anonymous reviewers for valuable advice on the manuscript. Ron van der Hulst provided helpful discussions and triggered the study of male sterility in dandelions.

\section{References}

Corriveau JL, Coleman AW (1988). Rapid screening method to detect potential biparental inheritance of plastid DNA and results for over 200 species. Am J Bot 75: 1443-1458. 
De Kovel CGF, De Jong G (2000). Selection on apomictic lineages of Taraxacum at establishment in a mixed sexualapomictic population. J Evol Biol 13: 561-568.

Den Nijs JCM, Sterk AA (1980). Cytogeographical studies of Taraxacum sect. Taraxacum (= sect. Vulgaria) in Central Europe. Bot Jahrb System 101: 527-554.

Den Nijs JCM, Sterk AA (1984). Cytogeography of Taraxacum sectio Taraxacum and sectio Alpestria in France and some adjecent parts of Italy and Switserland, including some taxonomic remarks. Act Bot Neerl 33: 1-24.

Douhovnikoff V, Dodd RS (2003). Intra-clonal variation and a similarity threshold for identification of clones: application to Salix exigua using AFLP molecular markers. Theor Appl Genet 106: 1307-1315.

Dumolin-Lapeque S, Pemonge M-H, Petit RJ (1997). An enlarged set of consensus primers for the study of organelle DNA in plants. Mol Ecol 6: 393-397.

Falque M, Keurentjes J, Bakx-Schotman JMT, van Dijk P (1998). Development and characterization of microsatellite markers in the sexual-apomictic complex Taraxacum officinale (dandelion). Theor Appl Genet 97: 283-292.

Gouyon PH, Couvet D (1987). A conflict between two sexes, females and hermaphrodites. In: Stearns SC (ed) The Evolution of Sex and its Consequences. Birkhäuser: Basel.

Hanson MR (1991). Plant mitochondrial mutations and male sterility. Annu Rev Genet 25: 461-486.

Hardy OJ, Vekemans X (2002). SPAGEDi: a versatile computer program to analyse spatial genetic structure at the individual or population levels. Mol Ecol Notes 2: 618-620.

Kirschner J, Stepanek J, Mes THM, den Nijs JCM, Oosterveld P, Storchova H et al (2003). Principal features of the cpDNA evolution in Taraxacum (Asteraceae, Lactuceae): a conflict with taxonomy. Plant Syst Evol 239: 231-255.

Kollmann J, Steinger T, Roy BA (2000). Evidence of sexuality in European Rubus (Rosaceae) species based on AFLP and allozyme analysis. Am J Bot 87: 1592-1598.

Malecka J (1971). Process of degeneration in anthers tapetum of two male-sterile species of Taraxacum. Genet Polonica 12: 301-303.

Manly BFJ (1991). Randomization and Monte Carlo Methods in Biology. Chapman \& Hall: London.

Maynard Smith J (1978). The Evolution of Sex. Cambridge University Press: Cambridge.

Meirmans PG (2005). Ecological and Genetic Interactions Between Diploid Sexual and Triploid Apomictic Dandelions. PhD Thesis. University of Amsterdam: Amsterdam.

Meirmans PG, Van Tienderen PH (2004). GENOTYPE and GENODIVE: two programs for the analysis of genetic diversity of asexual organisms. Mol Ecol Notes 4: 792-794.
Meirmans PG, Vlot EC, Den Nijs JCM, Menken SBJ (2003). Spatial ecological and genetic structure of a mixed population of sexual diploid and apomictic triploid dandelions. J Evol Biol 16: 343-352.

Menken SBJ, Smits E, den Nijs JCM (1995). Genetical population structure in plants: gene flow between diploid sexual and triploid asexual dandelions (Taraxacum section Ruderalia). Evolution 49: 1108-1118.

Morita T, Menken SBJ, Sterk AA (1990). Hybridization between European and Asian dandelions (Taraxacum sectio Ruderalia and section Mongolica). 1. Crossability and breakdown of self-incompatibility. N Phytol 114: 519-529.

Ó Foighil D, Smith MJ (1995). Evolution of asexuality in the cosmopolitan marine clam Lasnea. Evolution 49: 140-150.

Richards AJ (1986). Plant Breeding Systems. Allen \& Unwin: London.

Ronfort JL, Jenczewski E, Bataillon T, Rousset F (1998). Analysis of population structure in autotetraploid species. Genetics 150: 921-930.

Solbrig OT, Simpson BB (1974). Components of regulation of a population of dandelions in Michigan. J Ecol 62: 473-486.

Sterk AA (1987). Aspects of the population biology of sexual dandelions in the Netherlands. In: Huiskes AHL, Blom CWPM, Rozema J (eds) Vegetation Between Land and Sea. Junk: Dordrecht. pp 284-290.

Taberlet P, Gielly L, Pautou G, Bouvet J (1991). Universal primers for amplification of three non-coding regions of chloroplast DNA. Plant Mol Biol 17: 1105-1109.

Van der Hulst RGM, Meirmans P, Van Tienderen PH, Van Damme JMM (2004). Nuclear-cytoplasmic male-sterility in diploid dandelions. Heredity 93: 43-50.

Van der Hulst RGM, Mes THM, Falque M, Stam P, Den Nijs JCM, Bachmann K (2003). Genetic structure of a population sample of apomictic dandelions. Heredity 90: 326-335.

Van Dijk PJ (2003). Ecological and evolutionary opportunities of apomixis: insights from Taraxacum and Chondrilla. Philos Trans Roy Soc London Ser B-Biol Sci 358: 1113-1120.

Verduijn MH, Van Dijk PJ, Van Damme JMM (2004). The role of tetraploids in the sexual-asexual cycle in dandelions (Taraxacum). Heredity 93: 390-398.

Vrijenhoek RC (1979). Factors affecting clonal diversity and coexistence. Am Zool 19: 787-797.

Weinzierl RP, Berthold K, Beukeboom LW, Michiels NK (1998). Reduced male allocation in the parthenogenetic hermaphrodite Dugesia polychroa. Evolution 52: 109-115.

Wittzell H (1999). Chloroplast DNA variation and reticulate evolution in sexual and apomictic sections of dandelions. Mol Ecol 8: 2023-2035.

Supplementary Information accompanies the paper on the Heredity website (http://www.nature.com/hdy) 